\title{
COMPARATIVE STUDY ON ANALYSIS OF TELECOM TOWER USING INDIA AND AMERICAN STANDARDS
}

\section{Srinivas Tanuku ${ }^{*}$, Prof. K. Rama Mohana Rao $^{2}$ and B. Pandu Rangarao ${ }^{3}$}

1, Senior Manager - Tower Assessment, RAMBOLL India Private Limited, Hyderabad, India

2, Professor, Dept. of Civil Engineering, Jawaharlal Nehru Technological University, Hyderabad, Telangana, India.

${ }^{3}$ Research Scholar, JNTU College of Engineering, Hyderabad; General Manager in National Council for Cement and Building Material, New Delhi, India

${ }^{1}$ Email: srinit@ramboll.com*(Corresponding author)

${ }^{2}$ Email: rmkunapareddy@gmail.com

${ }^{3}$ Email: ncbtpqi@gmail.com

\begin{abstract}
Self-supporting lattice tower are being effective structural system by considering simple, light weight, easy fabrication and installation for supporting telecom equipment at elevated heights. With increase in demand of lattice towers, a critical review on approach for analysis is highly essential to ensure reliable and safe structures. In this paper, a comparative study is taken up on methodologies followed in both national standards (India, America) for assessment of wind loads on bare tower, linear accessories, discrete accessories along with design resistance of members and connections for Two different configurations - Square angular tower, Triangular Hybrid Tower. From the detailed analysis, it is concluded that, American standard (ANSI/TIA-222H) is using Ultimate windspeed for calculation of wind loads based on risk category of structure along with strength reduction factors based on criticality of components compared to Indian Standards (IS 875(Part 3)-2015, IS 802) which resulted lesser wind load on structure i.e., 30\% in Square Tower (Oblique wind direction) and 23\% in Triangular Hybrid Tower using ANSI/TIA-222H. Also, no major difference observed for calculation of member capacity and connection. Therefore, it is concluded that Tower weights approximately reduces by $10-15 \%$ based on Tower configuration using ANSI/TIA222H compared to Indian Standards
\end{abstract}

Keywords: Analysis, Hot-rolled steel angles, Self-supporting lattice towers, Wind loads.

https://doi.org/10.47412/DWHY3671

\section{Introduction}

Self-supporting lattice tower are being effective structural system by considering light weight, easy fabrication and installation and these are normally square or triangular in plan, made up of steel angle or hollow sections. Wind is predominant load for analysis of these slender structures, therefore in depth understanding on wind loads and buckling capacities of steel members are essential for structural analysis. In India, general wind loading standard is being referred for load calculations and member capacities are obtaining from specialized standards referring Transmission line towers. Using generic standards may lead to conservative approach, sometimes underestimating critical parameter which are affecting safety of structure. In this paper, an attempt 
is made to review current practice by comparing with American standards specially dealt on analysis of telecommunication towers and parametric study is undertaken with two different configurations - Square angular tower consists of Hot Rolled steel angle sections and Triangular Hybrid Tower comprises of Legs with Hollow steel sections and bracings are of steel angle sections. for comparative study on overall impact.

\section{Wind and Influencing Parameters}

Wind Means the motion of air in the atmosphere with respect to surface of the earth is fundamentally caused by variable solar heating of earth's atmosphere. The earth surface exerts on the moving air a horizontal drag force, whose effect is to retard the flow. This effect is diffused by turbulent mixing throughout a region referred as atmospheric boundary layer. The depth of boundary layer depending up on the wind intensity, roughness of terrain and angle of latitude. Within boundary layer, the wind speed increases with elevation its magnitude at the top of boundary layer is often referred to as a gradient speed. Therefore, parameter such as risk level of structure, terrain influences, topographical features, shape factor, direction factor and as well structural response to wind all are contributing wind effects on lattice towers.

\subsection{Basic Wind Speed}

Basic Wind speed is defined as the peak gust velocity averaged over a short time duration and corresponds to mean height above ground level in open terrain. Basic wind speed shall be extracted from respective country wind map, and duration of basic wind speed given in both codes i.e., IS 875 (Part 3), ANSI/TIA-222H observed as 3 second duration for a 50-year return period. In both codes, basic wind speed duration is remains same, however ultimate load factor based on higher return period (Predefined risk category of structure) is included in basic wind speed for obtaining ultimate wind speed in ANSI/TIA-222H with no extra load factors in analysis load combinations, whereas as per IS 875 (Part 3) - 2015, the nominal risk coefficient along with load factor of 1.5 is being considered in analysis load combination. Wind Speed against return period using two standards are listed below.

Table 2.1: Wind Speed Against Return Period

\begin{tabular}{|c|c|c|}
\hline $\begin{array}{c}\text { Return Period } \\
\text { (Years) }\end{array}$ & IS & 222 H \\
\hline 50 & 47 & 47 \\
\hline 5 & 33.4 & - \\
\hline 25 & 42.3 & - \\
\hline 100 & 50.3 & - \\
\hline 300 & - & 55.5 \\
\hline 700 & - & 59.2 \\
\hline 1700 & - & 63.9 \\
\hline 3000 & - & 66.3 \\
\hline
\end{tabular}




\subsection{Terrain and Height Multiplier}

The wind speed varies with height due to ground friction and amount of friction varies with ground roughness and is characterized by terrain / exposure categories based on surrounding obstruction. Three categories are defined in ANSI/TIA-222H against four categories in IS 875 (Part 3). An average of $4 \%, 10 \%$ values are increased at $50-100 \mathrm{~m}$ and $150-200 \mathrm{~m}$ height respectively in ANSI/TIA-222H compared to IS 875 (Part 3) for open terrain with scattered obstruction having heights less than $10 \mathrm{~m}$.

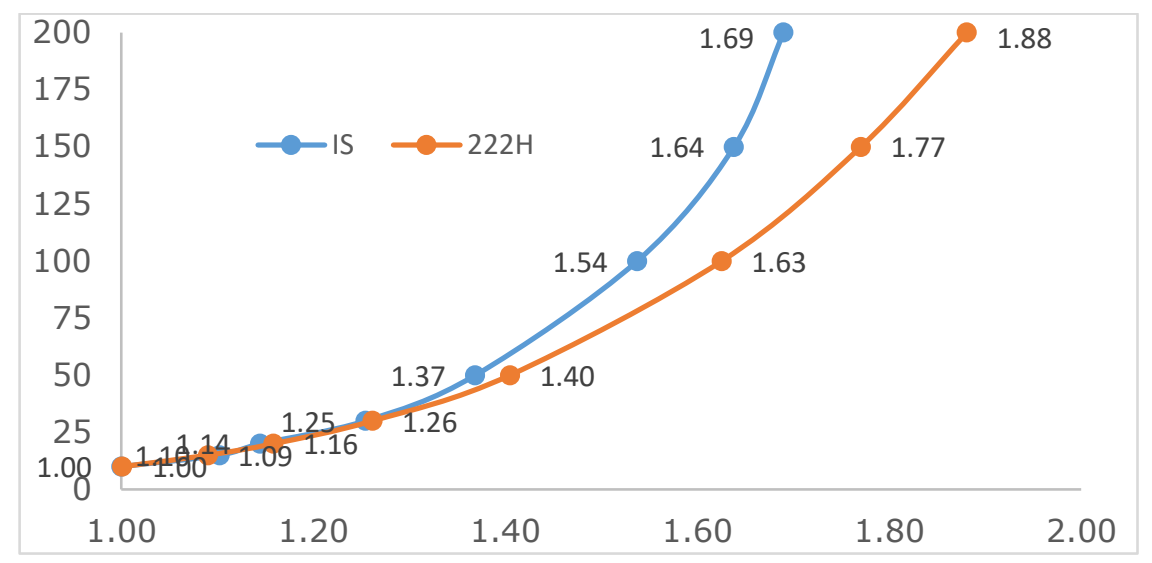

Fig. 2.1: Terrain Height Multiplier (Open Terrain)

\subsection{Topography Influences}

Topography influence that have some affects are - funnelling of winds (occurs when there is natural flow of air from an unrestricted area through restricted area, such as mountain pass), mountains (Flow over the crest of hill ridge when the wind is normal to edge is considerably less turbulent than the flow of upwind of hill and wind velocity is increased. The effect of topography is to accelerate wind near the summits of hills or crests or cliffs, escarpments or ridges and decelerate the wind in valleys or near the foot of cliffs, steep escarpment, or ridges. As per IS 875 (Part 3), detailed methodology is given for calculation of topographic factor based on up wind slop of hill / ridge with maximum factor of 1.36. In ANSI/TIA-222-H, four categories are defined based on structure location on hill and empirical formulae and rigorous calculations are given for topographic effects against each category without any maximum limits.

\subsection{Force Coefficient}

Force coefficient is the ratio of resulting force per unit area in the direction of wind to the applied wind pressure. It accounts for the effect of member characteristics (Shape, size, solidity, shielding and surface roughness) also it accounts for both wind ward and lee ward faces including shielding of leeward face by members in wind ward faces. Force coefficient is given for different type of tower configurations (Square / Equilateral triangular towers) and type of member such as Flat or tubular member based on frame solidity ratio (Ratio of Projected area of members and Total area of panel under considered). Reference to table 3.1, force coefficient is reduced by $9 \%$ and $4 \%$ for square and triangular tower respectively in ANSI/TIA-222G. 
Table 2.2: Force Coefficient - Tower with Flat Sections

\begin{tabular}{|c|c|c|c|c|}
\hline \multirow{2}{*}{$\begin{array}{c}\text { Solidity } \\
\text { Ratio }\end{array}$} & \multicolumn{2}{|c|}{ Square } & \multicolumn{2}{c|}{ Triangular } \\
\cline { 2 - 5 } & IS & $\mathbf{2 2 2 H}$ & IS & $\mathbf{2 2 2 H}$ \\
\hline 0.1 & 3.80 & 3.45 & 3.1 & 2.96 \\
\hline 0.2 & 3.30 & 2.98 & 2.7 & 2.60 \\
\hline 0.3 & 2.80 & 2.59 & 2.3 & 2.30 \\
\hline 0.4 & 2.30 & 2.28 & 1.9 & 2.06 \\
\hline 0.5 & 2.10 & 2.05 & 1.5 & 1.90 \\
\hline
\end{tabular}

Table 2.3: Force Coefficient - Flat Appurtenances

\begin{tabular}{|c|c|c|}
\hline Aspect Ratio & IS & $\mathbf{2 2 2}$ H \\
\hline$\leq 2.5$ & 1.25 & 1.2 \\
\hline 7 & 1.34 & 1.4 \\
\hline$\geq 25$ & 1.65 & 2.0 \\
\hline
\end{tabular}

\subsection{Structural Response to Wind}

Wind force Is essentially dynamic in nature even through it is treated as steady force for simplicity in analysis. Due to turbulent nature of wind velocities, the wind loads acting on structure also highly fluctuating. The back-ground response made up of largely low-frequency contribution below the lowest natural frequency of vibration is the largest contributor for along wind loading. The resonant contribution becomes more significant, will eventually dominate as structure becomes taller in relation to their width and their natural frequencies becomes lower. The resonant response will be significant when the structure frequency is less than $1.0 \mathrm{~Hz}$. When structure experiences resonant dynamic response, counteracting structural forces come in to play to balance wind forces are - inertia force proportional to mass of structure, damping or energy absorbing force, elastic or stiffness force proportional to deflection. As per IS 875 (Part 3), flexible slender structures (defined as first mode frequency is less than $1.0 \mathrm{~Hz}$ ) shall be investigated to ascertain the importance of wind induced oscillations or excitations along wind and across wind directions. Static wind loading is recommended for rigid structure (first mode frequency is greater than 1.0 $\mathrm{Hz}$ ), where maximum self-supporting lattice towers are fall in this category i.e., rigid structure by considering first mode frequency. As per ANSI/TIA-222H, a factor of $0.85-1.00$ is recommended as gust effect factor which accounts for loading effects in the along-wind direction due to wind turbulence - structure interaction for self-supporting structure (Rigid structure) which shall be multiplied to ultimate design wind pressure obtained from ultimate wind speed of 3 second duration. While deciding frequency of structure, separate empirical formula is given in ANSI Code which can be reasonably estimate frequency of lattice towers compared to generic formula given in IS 875 (Part 3).

\subsection{Design Wind Pressure}

The design wind pressure at any height above mean ground level shall be calculated as per below against each standard. 
As per IS 875 (Part 3) - 2015, design wind pressure is given by,

$=\left[0.6\left(\mathrm{~V}_{\mathrm{b}} \mathrm{K}_{1} \mathrm{~K}_{2} \mathrm{~K}_{3} \mathrm{~K}_{4}\right)^{2}\right] \times \mathrm{K}_{\mathrm{d}} \mathrm{K}_{\mathrm{a}} \mathrm{K}_{\mathrm{c}}$

$\rho_{z}$

As per ANSI/TIA-222H, design wind pressure is given by,

$\rho_{\mathrm{z}}=0.613 \mathrm{~K}_{\mathrm{z}} \mathrm{K}_{\mathrm{zt}} \mathrm{K}_{\mathrm{d}} \mathrm{K}_{\mathrm{s}} \mathrm{K}_{\mathrm{e}} \mathrm{V}^{2}$

\section{2}

Ultimate Design wind pressure (with Load factors, 1.5 for IS $875 \& 1.0$ for ANSI/TIA-222H) including force coefficient (Solidity ratio assumed as 0.2 ) using both standards are summarized in Fig. 4.1 and 4.2.

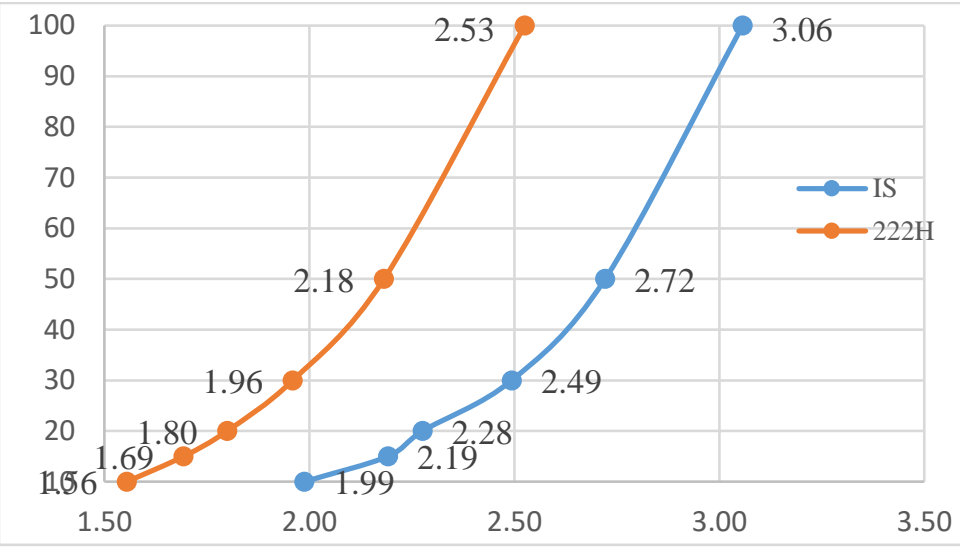

Fig. 2.2: Design Wind Pressure, $\mathrm{kN} / \mathrm{m}^{2}$ (Face Wind)

Lattice structures are analysed almost exclusively as ideal elastic three-dimensional trusses made up of straight members and pin-connected at joints which produces - only joint displacements, tension and compression in the members. The flexural buckling strength of compression members is being derived from Euler's buckling theory and is mainly depends on slenderness ratio and vary based on members - concentric, eccentric. In both codes, the capacity calculation remains same except variation on strength reduction factors. Therefore, detailed methodology is not presented in this sec Fig. 4.1: Design Wind Pressure, $\mathrm{kN} / \mathrm{m}^{2}$ capacit (Face Wind)

Fog. 4.2: Design Wind Pressure, $\mathrm{kN} / \mathrm{m}^{2} \quad$ rection (Corner Wind)

\section{Parametric Study}

Two Different configuration (Square - Composed of Angle sections, Triangular - Legs with pipes and others with angular bracings) are considered for parametric study for a basic wind speed of $47 \mathrm{~m} / \mathrm{s}$ ( 3 second gust) with open terrain with well scattered obstruction of $1.5-10 \mathrm{~m}$. Basic Tower details are listed below along with tower elevation drawing. 
Table 4.1: Basic Tower Details

\begin{tabular}{c|lcc}
\hline$\#$ & \multicolumn{1}{|c}{ Description } & Case 1 & Case 2 \\
\hline $\mathrm{a}$ & Tower Configuration & Square & Triangular \\
$\mathrm{b}$ & Legs & Steel Angles & Steel - CHS \\
$\mathrm{c}$ & Bracings & Steel Angles & Steel Angles \\
$\mathrm{d}$ & Bottom Face Width & $7.35 \mathrm{~m}$ & $9.15 \mathrm{~m}$ \\
$\mathrm{e}$ & Top Face Width & $1.80 \mathrm{~m}$ & $2.00 \mathrm{~m}$ \\
$\mathrm{f}$ & Vertical Portion & $15 \mathrm{~m}$ & $10 \mathrm{~m}$ \\
\hline
\end{tabular}

Table 4.2: Wind Influence Parameters

\begin{tabular}{c|lcc}
\hline$\#$ & \multicolumn{1}{c}{ Description } & IS & ANSI \\
\hline $\mathrm{a}$ & Basic Wind Speed (3Sec.) & $47 \mathrm{~m} / \mathrm{s}$ & $47 \mathrm{~m} / \mathrm{s}$ \\
$\mathrm{b}$ & Risk Coefficient & 1.00 & 1.26 \\
$\mathrm{c}$ & Topography factor & 1.00 & 1 \\
$\mathrm{~d}$ & Ultimate Wind Speed & $47 \mathrm{~m} / \mathrm{s}$ & $59.2 \mathrm{~m} / \mathrm{s}$ \\
$\mathrm{e}$ & Basic Design wind Pressure & 135.153 & 158.38 \\
& $\left(\mathrm{~kg} / \mathrm{m}^{2}\right)$ & & \\
$\mathrm{f}$ & Load factor & 1.5 & 1.0 \\
$\mathrm{~g}$ & Ultimate Design Wind & 202.73 & 158.38 \\
& Pressure $\left(\mathrm{kg} / \mathrm{m}^{2}\right)$ & & \\
\hline
\end{tabular}

Table 4.3: Antenna Details

\begin{tabular}{l|l|c}
\hline \multicolumn{2}{c|}{ Antenna Type } & Square / Triangular \\
\hline \multirow{2}{*}{ GSM Antenna } & Size (m) & $2.58 \times 0.262 \times 0.116$ \\
\cline { 2 - 3 } & Quantity (Nos) & 12 \\
\hline \multirow{2}{*}{ MW Antenna } & Size (m) & $1.2 \mathrm{~m}$ Dia. \\
\cline { 2 - 3 } & Quantity (Nos) & 3 \\
\hline
\end{tabular}




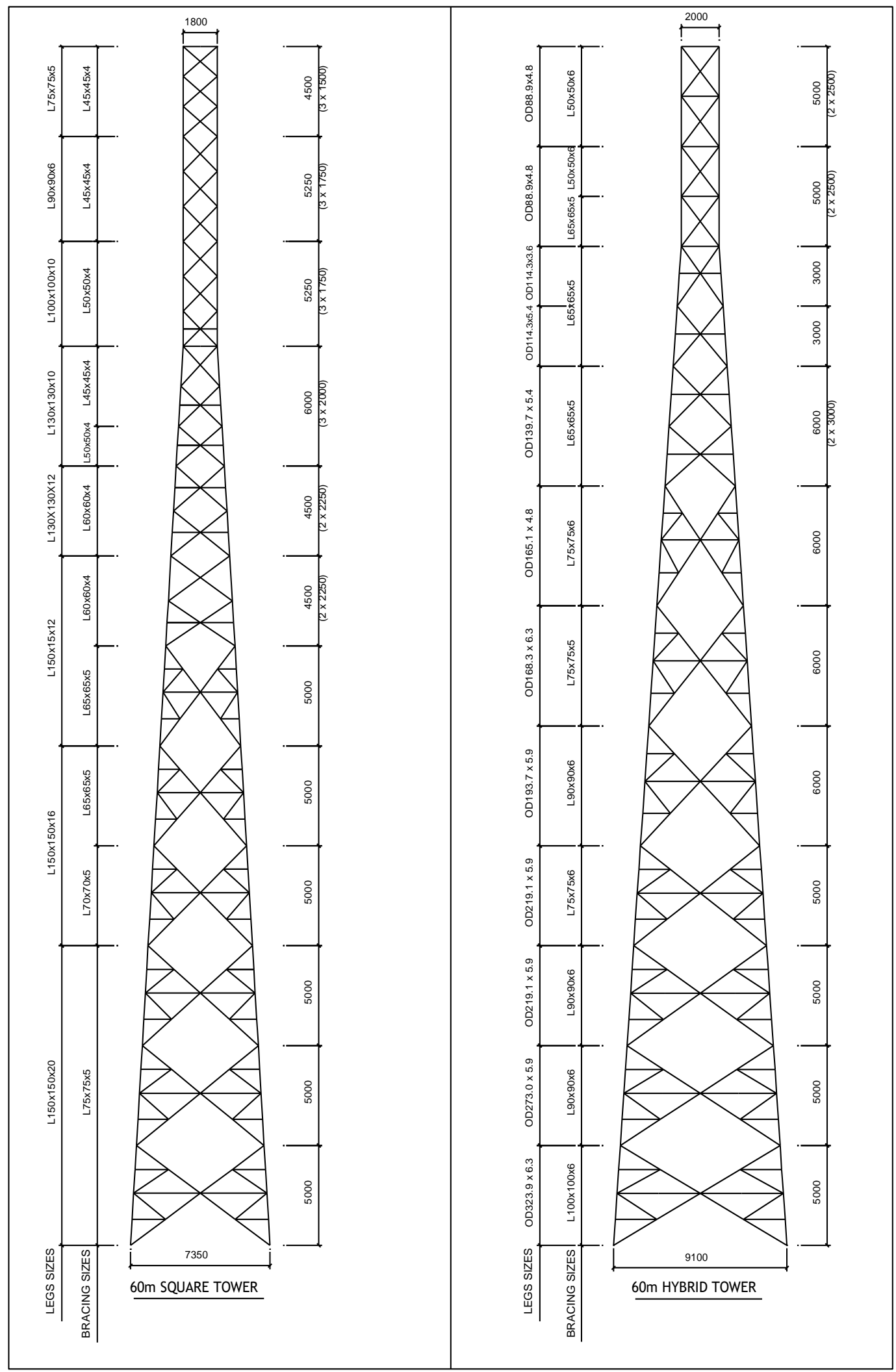

Fig. 4.1: Tower Elevation Drawing - Both Options 


\subsection{Wind Load}

Section wise wind loads are calculated on tower body for two different configurations considered in the parametric study and results are tabulated as below.

Table 4.5: Section Wise Wind Load Comparison - Square Tower

\begin{tabular}{|c|c|c|c|c|c|c|c|c|c|c|c|}
\hline \multirow{2}{*}{$\begin{array}{l}\text { Sectio } \\
\text { n Ht. } \\
(\mathrm{m})\end{array}$} & \multirow{2}{*}{$\begin{array}{l}\text { Cum } \\
. \mathrm{Ht} \\
(\mathrm{m})\end{array}$} & \multirow{2}{*}{$\begin{array}{c}\text { Expose } \\
\text { d Area } \\
\left(\mathrm{m}^{2}\right)\end{array}$} & \multirow{2}{*}{$\begin{array}{c}\text { Solidit } \\
\text { y } \\
\text { Ratio } \\
(\Phi)\end{array}$} & \multicolumn{2}{|c|}{ Cf - Angles } & \multicolumn{2}{|c|}{$\begin{array}{l}\text { Design } \\
\text { Wind } \\
\text { Pressure }\end{array}$} & \multicolumn{2}{|c|}{$\begin{array}{c}\text { Ultimate } \\
\text { Wind Load } \\
(\mathrm{kN})\end{array}$} & \multicolumn{2}{|c|}{$\begin{array}{c}\text { Ultimate } \\
\text { Wind Load } \\
(\mathbf{k N})\end{array}$} \\
\hline & & & & IS & 222 & IS & 222 & IS & $222 \mathrm{H}$ & IS & $222 \mathrm{H}$ \\
\hline 4.5 & 60.0 & 1.42 & 0.17 & 3.43 & 3.09 & 1.8 & 2.25 & 13.4 & 9.82 & 16.0 & 11.12 \\
\hline 5.25 & 55.5 & 1.62 & 0.17 & 3.44 & 3.10 & 1.8 & 2.21 & 15.3 & 11.12 & 18.3 & 12.56 \\
\hline 5.25 & 50.2 & 1.88 & 0.20 & 3.30 & 2.98 & 1.8 & 2.16 & 16.7 & 12.12 & 20.0 & 13.94 \\
\hline 6 & 45.0 & 2.71 & 0.21 & 3.25 & 2.94 & 1.7 & 2.10 & 23.1 & 16.75 & 27.7 & 19.37 \\
\hline 4.5 & 39.0 & 2.31 & 0.18 & 3.39 & 3.06 & 1.7 & 2.05 & 20.0 & 14.42 & 24.0 & 16.39 \\
\hline 4.5 & 34.5 & 2.54 & 0.17 & 3.47 & 3.13 & 1.6 & 1.99 & 22.1 & 15.75 & 26.5 & 17.73 \\
\hline 5 & 30.0 & 3.03 & 0.15 & 3.53 & 3.19 & 1.6 & 1.93 & 26.0 & 18.60 & 31.3 & 20.74 \\
\hline 5 & 25.0 & 3.17 & 0.14 & 3.61 & 3.26 & 1.5 & 1.85 & 26.6 & 19.05 & 31.9 & 21.03 \\
\hline 5 & 20.0 & 3.44 & 0.13 & 3.64 & 3.29 & 1.4 & 1.75 & 27.9 & 19.81 & 33.5 & 21.77 \\
\hline 5 & 15.0 & 3.78 & 0.13 & 3.65 & 3.30 & 1.3 & 1.63 & 28.7 & 20.33 & 34.5 & 22.32 \\
\hline 5 & 10.0 & 3.98 & 0.12 & 3.68 & 3.33 & 1.3 & 1.47 & 29.1 & 19.43 & 34.9 & 21.23 \\
\hline 5 & 5.00 & 4.26 & 0.12 & 3.69 & 3.34 & 1.3 & 1.32 & 31.3 & 18.83 & 37.5 & 20.54 \\
\hline
\end{tabular}

Table 4.6: Section Wise Wind Load Comparison - Triangular Tower

\begin{tabular}{|c|c|c|c|c|c|c|c|c|c|c|c|c|}
\hline \multirow{2}{*}{$\begin{array}{c}\text { Sectio } \\
\text { n Ht. } \\
(\mathrm{m})\end{array}$} & \multirow{2}{*}{$\begin{array}{l}\mathrm{Cu} \\
\mathrm{m} . \\
\mathrm{Ht}\end{array}$} & \multicolumn{2}{|c|}{$\begin{array}{c}\text { Exposed } \\
\text { Area }\left(\mathrm{m}^{2}\right)\end{array}$} & \multirow{2}{*}{$\begin{array}{c}\text { Solidit } \\
\text { y } \\
\text { Ratio }\end{array}$} & \multicolumn{2}{|c|}{ Cf - Pipes } & \multicolumn{2}{|c|}{$\begin{array}{c}\text { Cf - } \\
\text { Angles }\end{array}$} & \multicolumn{2}{|c|}{$\begin{array}{c}\text { Design } \\
\text { Wind }\end{array}$} & \multicolumn{2}{|c|}{$\begin{array}{c}\text { Ultimate } \\
\text { Wind Load }\end{array}$} \\
\hline & & Pipe & Angle & & IS & 222 & IS & 222 & IS & 222 & IS & 222H \\
\hline 5 & 60 & 0.89 & 0.79 & 0.17 & 1.6 & 1.47 & 2.8 & 2.71 & 1.8 & 2.25 & 10.1 & 7.73 \\
\hline 5 & 55 & 0.89 & 0.88 & 0.18 & 1.6 & 1.46 & 2.7 & 2.68 & 1.8 & 2.20 & 10.6 & 8.04 \\
\hline 3 & 50 & 0.69 & 0.67 & 0.20 & 1.6 & 1.32 & 2.6 & 2.58 & 1.8 & 2.17 & 7.85 & 5.73 \\
\hline 3 & 47 & 0.69 & 0.64 & 0.17 & 1.6 & 1.35 & 2.8 & 2.71 & 1.7 & 2.14 & 7.82 & 5.69 \\
\hline 6 & 44 & 1.68 & 1.45 & 0.16 & 0.9 & 1.23 & 2.8 & 2.74 & 1.7 & 2.09 & 15.1 & 12.66 \\
\hline 6 & 38 & 1.99 & 7 & 0.16 & 0.9 & 1.16 & 2.8 & 2.75 & 1.7 & 2.03 & 8.6 & 15.10 \\
\hline 6 & 32 & 2.02 & 2.11 & 0.14 & 0.9 & 1.16 & 2.9 & 2.82 & 1.6 & 1.95 & 19.9 & 16.14 \\
\hline 6 & 26 & 2.33 & 2.69 & 0.14 & 0.9 & 1.16 & 2.9 & 2.80 & 1.5 & 1.85 & 23.5 & 18.99 \\
\hline 5 & 20 & 2.20 & 2.55 & 0.14 & 0.9 & 1.16 & 2.9 & 2.80 & 1.4 & 1.75 & 21.2 & 16.94 \\
\hline 5 & 15 & 2.20 & 3.02 & 0.14 & 0.9 & 1.16 & 2.9 & 2.80 & 1.3 & 1.63 & 22.8 & 17.97 \\
\hline 5 & 10 & 2.74 & 3.45 & 0.15 & 0.9 & 1.16 & 2.8 & 2.76 & 1.3 & 1.47 & 25.0 & 18.61 \\
\hline 5 & 5 & 3.25 & 3.96 & 0.16 & 0.9 & 1.16 & 2.8 & 2.72 & 1.3 & 1.32 & 28.7 & 19.18 \\
\hline
\end{tabular}


Faculty of Engineering, The UWI, St. Augustine | June $1^{\text {st }}-5^{\text {th }}, 2020$

Table 4.7: Antenna Wind Load Comparison

\begin{tabular}{|c|c|c|c|}
\hline \multirow{7}{*}{$\begin{array}{c}\text { GSM } \\
\text { Antenna }\end{array}$} & Descrintion & IS & $222 \mathrm{H}$ \\
\hline & Area $\left(\mathrm{m}^{2}\right)$ & 0.68 & 0.68 \\
\hline & Elevation $(\mathrm{m})$ & 57.75 & 57.75 \\
\hline & Force Coefficient & 1.389 & 1.495 \\
\hline & Antenna - Otv & 12 & 12 \\
\hline & Total EPA $\left(\mathrm{m}^{2}\right)$ & 11.27 & 12.13 \\
\hline & Ultimate Wind Load & 31.27 & 27.54 \\
\hline \multirow{7}{*}{$\begin{array}{c}\text { MW } \\
\text { Antenna } \\
\text { (With } \\
\text { Radome) }\end{array}$} & Diameter $(\mathrm{m})$ & 1.2 & 1.2 \\
\hline & Elevation (m) & 57.75 & 57.75 \\
\hline & Force Coefficient & 1.2 & 0.863 \\
\hline & EPA / Antenna & 1.36 & 0.98 \\
\hline & Antenna - Otv & 3 & 3 \\
\hline & Total EPA $\left(\mathrm{m}^{2}\right)$ & 4.07 & 2.93 \\
\hline & Ultimate Wind Load & 11.29 & 6.65 \\
\hline
\end{tabular}

\subsection{Analysis}

Three-dimensional space truss analysis is carried out using STAAD-Pro V8 software by applying wind loads on different panels on complete tower. Wind forces due to tower body are distributed to the all sectional points at an elevation equally with fact that the force coefficient has accounted for both wind ward and leeward tower faces including shielding effects. And Wind forces due to linear, discrete accessories are distributed based on their location with respect tower centre of axis. Two wind directions are assumed - face wind (parallel to the frame) and corner wind in the analysis based on tower configuration considered for parametric study.
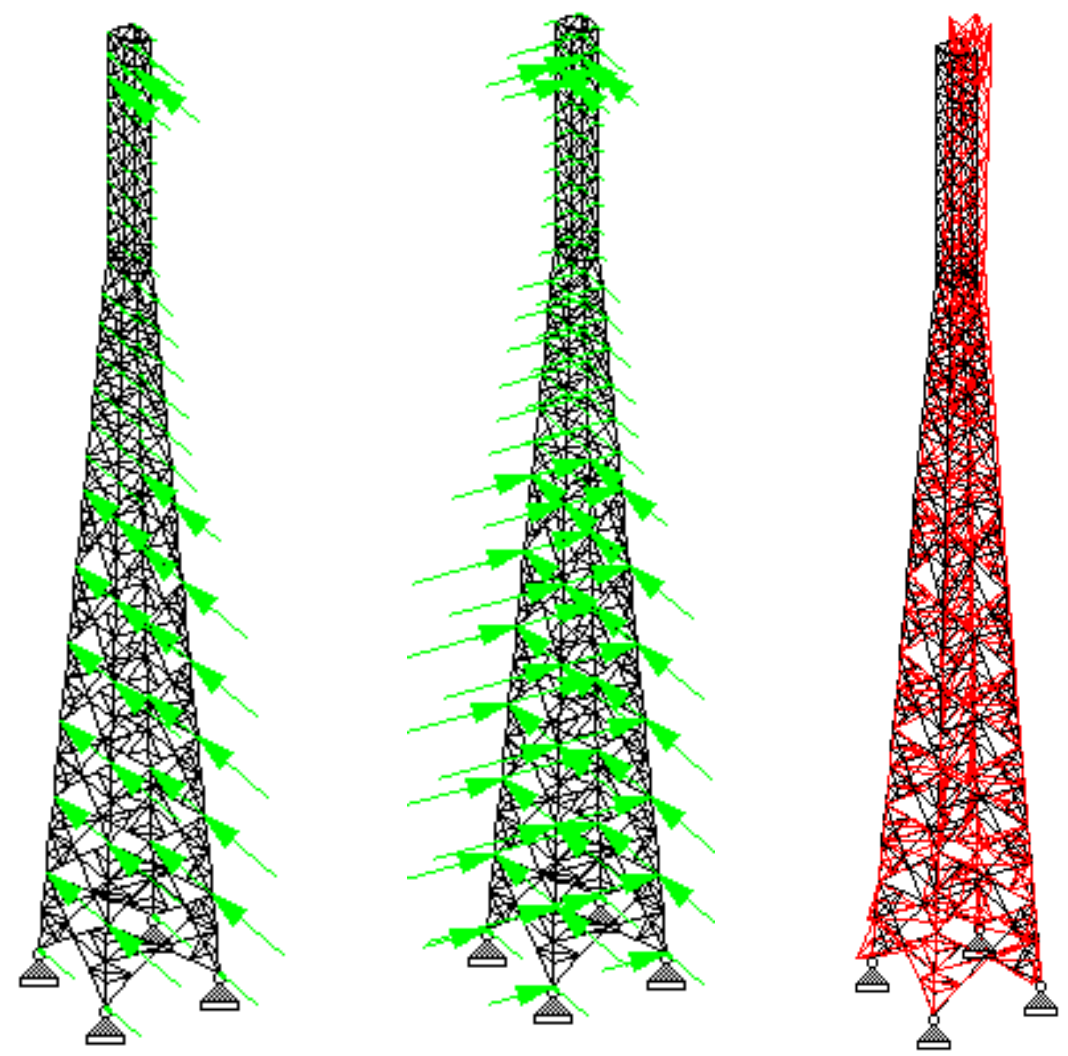

Fig. 4.2: Analysis Model of Square Tower - a) Face Wind, b) Diagonal Wind, c) Deflected Profile 


\subsection{Design Resistance}

Member design resistance are calculated based on two national standards against each section for two different configuration and are tabulated below.

Table 4.8: Section Wise Main leg member capacity Comparison - Square Tower

\begin{tabular}{|c|c|c|c|c|c|c|c|c|c|c|c|c|}
\hline \multirow{2}{*}{$\begin{array}{l}\text { Sec. } \\
\text { No. }\end{array}$} & \multirow{2}{*}{$\begin{array}{l}\text { Secti } \\
\text { on } H \\
\text { t. }(\mathrm{m})\end{array}$} & \multirow{2}{*}{$\begin{array}{c}\text { Cum } \\
. \mathrm{Ht}\end{array}$} & \multicolumn{5}{|c|}{ Section Properties (mm) } & \multicolumn{2}{|c|}{ Properties } & \multirow{2}{*}{$\begin{array}{l}\text { Eff.L } \\
(\mathrm{mm})\end{array}$} & \multicolumn{2}{|c|}{$\begin{array}{c}\text { Member } \\
\text { Capacity } \\
(\mathrm{kN})\end{array}$} \\
\hline & & & $\begin{array}{l}\text { Ty } \\
\text { pe }\end{array}$ & $\begin{array}{l}\text { Wi } \\
\text { dth }\end{array}$ & $\begin{array}{c}\text { Dep } \\
\text { th }\end{array}$ & $\begin{array}{l}\text { Th } \\
\text { k. }\end{array}$ & $\begin{array}{c}\text { Fy } \\
(\mathrm{Mpa} \\
\text { ) }\end{array}$ & $\underset{\left(\mathrm{mm}^{2}\right)}{\mathrm{Ag}}$ & $\begin{array}{c}\mathrm{R} \\
(\mathrm{mm})\end{array}$ & & IS & $\begin{array}{c}222 \\
\text { H }\end{array}$ \\
\hline $\begin{array}{c}\text { See- } \\
12\end{array}$ & 4.50 & $\begin{array}{c}60.0 \\
0\end{array}$ & $\mathrm{~L}$ & 75 & 75 & 5 & 250 & 725 & 14.9 & 1500 & 121 & 123 \\
\hline $\begin{array}{c}\text { Sec- } \\
11\end{array}$ & 5.25 & $\begin{array}{c}55.5 \\
0\end{array}$ & $\mathrm{~L}$ & 90 & 90 & 6 & 250 & 1044 & 17.9 & 1750 & 178 & 182 \\
\hline $\begin{array}{c}\text { Sec- } \\
10\end{array}$ & 5.25 & $\begin{array}{c}50.2 \\
5\end{array}$ & $\mathrm{~L}$ & 100 & 100 & 10 & 250 & 1900 & 19.7 & 1750 & 386 & 386 \\
\hline Sec-9 & 6.00 & $\begin{array}{c}45.0 \\
0\end{array}$ & $\mathrm{~L}$ & 130 & 130 & 10 & 250 & 2500 & 25.7 & 2010 & 562 & 562 \\
\hline Sec-8 & 4.50 & $\begin{array}{c}39.0 \\
0\end{array}$ & $\mathrm{~L}$ & 130 & 130 & 12 & 250 & 2976 & 25.6 & 1190 & 694 & 694 \\
\hline Sec-7 & 4.50 & $\begin{array}{c}34.5 \\
0\end{array}$ & $\mathrm{~L}$ & 150 & 150 & 12 & 250 & 3456 & 29.6 & 2260 & 763 & 763 \\
\hline Sec-6 & 5.00 & $\begin{array}{c}30.0 \\
0\end{array}$ & $\mathrm{~L}$ & 150 & 150 & 12 & 250 & 3456 & 29.6 & 1360 & 806 & 806 \\
\hline Sec-5 & 5.00 & $\begin{array}{c}25.0 \\
0\end{array}$ & $\mathrm{~L}$ & 150 & 150 & 16 & 250 & 4544 & 29.4 & 1340 & 1061 & 1061 \\
\hline Sec-4 & 5.00 & $\begin{array}{c}20.0 \\
0\end{array}$ & $\mathrm{~L}$ & 150 & 150 & 16 & 250 & 4544 & 29.4 & 1330 & 1062 & 1062 \\
\hline Sec-3 & 5.00 & $\begin{array}{c}15.0 \\
0\end{array}$ & $\mathrm{~L}$ & 150 & 150 & 20 & 240 & 5600 & 29.3 & 1320 & 1261 & 1261 \\
\hline Sec-2 & 5.00 & $\begin{array}{c}10.0 \\
0\end{array}$ & $\mathrm{~L}$ & 150 & 150 & 20 & 240 & 5600 & 29.3 & 1320 & 1261 & 1261 \\
\hline Sec-1 & 5.00 & 5.00 & $\mathrm{~L}$ & 150 & 150 & 20 & 240 & 5600 & 29.3 & 1310 & 1262 & 1262 \\
\hline
\end{tabular}


Table 4.9: Section Wise Bracing member capacity Comparison - Square Tower

\begin{tabular}{|c|c|c|c|c|c|c|c|c|c|c|c|c|}
\hline \multirow{2}{*}{$\begin{array}{l}\text { Sec. } \\
\text { No. }\end{array}$} & \multirow{2}{*}{$\begin{array}{l}\text { Secti } \\
\text { on H } \\
\text { t. }(\mathrm{m})\end{array}$} & \multirow{2}{*}{$\begin{array}{c}\text { Cum } \\
. \mathrm{Ht}\end{array}$} & \multicolumn{5}{|c|}{ Section Properties (mm) } & \multicolumn{2}{|c|}{ Properties } & \multirow{2}{*}{$\begin{array}{l}\text { Eff. } \\
\text { Le. } \\
(\mathrm{mm})\end{array}$} & \multicolumn{2}{|c|}{$\begin{array}{c}\text { Member } \\
\text { Capacity }(\mathrm{kN})\end{array}$} \\
\hline & & & $\begin{array}{c}\mathrm{T} \\
\mathrm{y} \\
\mathrm{pe}\end{array}$ & $\begin{array}{l}\text { Wi } \\
\text { dth }\end{array}$ & $\begin{array}{l}\text { De } \\
\text { pth }\end{array}$ & $\begin{array}{l}\mathrm{T} \\
\mathrm{h} \\
\mathrm{k} .\end{array}$ & $\begin{array}{c}\text { Fy } \\
(\mathrm{mpa} \\
\text { ) }\end{array}$ & $\underset{\left(\mathrm{mm}^{2}\right)}{\mathrm{Ag}}$ & $\begin{array}{c}\mathrm{R} \\
(\mathrm{mm})\end{array}$ & & IS & $222 \mathrm{H}$ \\
\hline $\begin{array}{c}\text { See- } \\
12\end{array}$ & 4.50 & $\begin{array}{c}60.0 \\
0\end{array}$ & $\mathrm{~L}$ & 45 & 45 & 4 & 250 & 344 & 8.9 & 1170 & 41 & 41 \\
\hline $\begin{array}{c}\text { Sec- } \\
11\end{array}$ & 5.25 & $\begin{array}{c}55.5 \\
0\end{array}$ & $\mathrm{~L}$ & 45 & 45 & 4 & 250 & 344 & 8.9 & 1260 & 36 & 37 \\
\hline $\begin{array}{c}\text { Sec- } \\
10\end{array}$ & 5.25 & $\begin{array}{c}50.2 \\
5\end{array}$ & $\mathrm{~L}$ & 50 & 50 & 4 & 250 & 384 & 9.9 & 1260 & 48 & 48 \\
\hline Sec-9 & 6.00 & $\begin{array}{c}45.0 \\
0\end{array}$ & $\mathrm{~L}$ & 45 & 45 & 4 & 250 & 344 & 8.9 & 1480 & 28 & 28 \\
\hline Sec-8 & 4.50 & $\begin{array}{c}39.0 \\
0\end{array}$ & $\mathrm{~L}$ & 60 & 60 & 4 & 250 & 464 & 11.9 & 1840 & 41 & 41 \\
\hline Sec-7 & 4.50 & $\begin{array}{c}34.5 \\
0\end{array}$ & $\mathrm{~L}$ & 60 & 60 & 4 & 250 & 464 & 11.9 & 2060 & 34 & 34 \\
\hline Sec-6 & 5.00 & $\begin{array}{c}30.0 \\
0\end{array}$ & $\mathrm{~L}$ & 65 & 65 & 5 & 250 & 625 & 20.2 & 3450 & 49 & 49 \\
\hline Sec-5 & 5.00 & $\begin{array}{c}25.0 \\
0\end{array}$ & $\mathrm{~L}$ & 65 & 65 & 5 & 250 & 625 & 20.2 & 3630 & 45 & 45 \\
\hline Sec-4 & 5.00 & $\begin{array}{c}20.0 \\
0\end{array}$ & $\mathrm{~L}$ & 70 & 70 & 5 & 250 & 675 & 21.8 & 3820 & 51 & 51 \\
\hline Sec-3 & 5.00 & $\begin{array}{c}15.0 \\
0\end{array}$ & $\mathrm{~L}$ & 75 & 75 & 5 & 250 & 725 & 23.4 & 4040 & 56 & 56 \\
\hline Sec-2 & 5.00 & $\begin{array}{c}10.0 \\
0\end{array}$ & $\mathrm{~L}$ & 75 & 75 & 5 & 250 & 725 & 23.4 & 3880 & 60 & 60 \\
\hline Sec-1 & 5.00 & 5.00 & $\mathrm{~L}$ & 75 & 75 & 5 & 250 & 725 & 14.9 & 2260 & 69 & 69 \\
\hline
\end{tabular}


Table 4.10: Section Wise Main leg member capacity Comparison - Triangular Tower

\begin{tabular}{|c|c|c|c|c|c|c|c|c|c|}
\hline \multicolumn{9}{|c|}{ Section Properties } & \multicolumn{2}{c}{$\begin{array}{c}\text { Eff. } \\
\text { Len. } \\
(\mathrm{mm})\end{array}$} & $\begin{array}{c}\text { Member } \\
\text { Capacity }(\mathrm{kN})\end{array}$ \\
\cline { 1 - 1 } No. & Type & $\begin{array}{c}\text { Dia. } \\
(\mathrm{m})\end{array}$ & $\begin{array}{c}\text { Thk. } \\
(\mathrm{mm})\end{array}$ & $\begin{array}{c}\text { Fy } \\
(\mathrm{mpa})\end{array}$ & $\begin{array}{c}\mathrm{A}_{\mathrm{g}} \\
\left(\mathrm{mm}^{2}\right)\end{array}$ & $\mathrm{R}(\mathrm{mm})$ & $\mathbf{2 2 2 H}$ \\
\hline $\begin{array}{c}\text { See- } \\
12\end{array}$ & OD & 0.089 & 4.85 & 240 & 1281 & 29.80 & 2500 & 215 & 208 \\
\hline $\begin{array}{c}\text { Sec- } \\
11\end{array}$ & OD & 0.089 & 4.85 & 240 & 1281 & 29.80 & 2500 & 215 & 208 \\
\hline $\begin{array}{c}\text { Sec- } \\
10\end{array}$ & OD & 0.114 & 3.65 & 240 & 1269 & 39.10 & 1660 & 261 & 270 \\
\hline Sec-9 & OD & 0.114 & 5.40 & 240 & 1847 & 38.50 & 1630 & 381 & 392 \\
\hline Sec-8 & OD & 0.140 & 5.40 & 240 & 2278 & 47.50 & 1610 & 485 & 500 \\
\hline Sec-7 & OD & 0.165 & 4.85 & 240 & 2442 & 56.70 & 1660 & 529 & 544 \\
\hline Sec-6 & OD & 0.168 & 6.30 & 240 & 3206 & 57.30 & 1640 & 696 & 716 \\
\hline Sec-5 & OD & 0.194 & 5.90 & 240 & 3481 & 66.40 & 1620 & 767 & 786 \\
\hline Sec-4 & OD & 0.219 & 5.90 & 240 & 3952 & 75.40 & 1320 & 893 & 906 \\
\hline Sec-3 & OD & 0.219 & 5.90 & 240 & 3952 & 75.40 & 1310 & 893 & 906 \\
\hline Sec-2 & OD & 0.273 & 5.90 & 240 & 4951 & 94.50 & 1310 & 1133 & 1141 \\
\hline Sec-1 & OD & 0.324 & 6.30 & 240 & 6286 & 112.30 & 1300 & 1450 & 1453 \\
\hline
\end{tabular}

Table 4.11: Section Wise Bracing member capacity Comparison - Triangular Tower

\begin{tabular}{|c|c|c|c|c|c|c|c|c|c|c|c|c|}
\hline \multirow{2}{*}{$\begin{array}{l}\text { Sec. } \\
\text { No. }\end{array}$} & \multirow{2}{*}{$\begin{array}{c}\text { Secti } \\
\text { on } \\
\text { Ht. } \\
(\mathrm{m})\end{array}$} & \multirow{2}{*}{$\begin{array}{l}\mathrm{Cu} \\
\mathrm{m} . \\
\mathrm{Ht}\end{array}$} & \multicolumn{7}{|c|}{ Section Properties (mm) } & \multirow{2}{*}{$\begin{array}{l}\text { Eff. } \\
\text { Len. } \\
(\mathrm{mm})\end{array}$} & \multicolumn{2}{|c|}{$\begin{array}{c}\text { Member } \\
\text { Capacity }(\mathrm{kN})\end{array}$} \\
\hline & & & $\begin{array}{c}\text { Typ } \\
\text { e }\end{array}$ & $\begin{array}{l}\text { Wid } \\
\text { th }\end{array}$ & $\begin{array}{l}\text { De } \\
\text { pth }\end{array}$ & $\begin{array}{l}\mathrm{T} \\
\mathrm{h} \\
\mathrm{k} .\end{array}$ & $\begin{array}{c}\text { Fy } \\
(\mathrm{mpa})\end{array}$ & $\begin{array}{c}\mathrm{Ag}_{\mathrm{g}} \\
\left(\mathrm{mm}^{2}\right. \\
)\end{array}$ & $\begin{array}{c}\mathrm{R} \\
(\mathrm{mm} \\
)\end{array}$ & & IS & $222 \mathrm{H}$ \\
\hline $\begin{array}{c}\text { See- } \\
12\end{array}$ & 5 & 60 & $\mathrm{~L}$ & 50 & 50 & 6 & 250 & 564 & 9.8 & 1600 & 42 & 42 \\
\hline $\begin{array}{c}\text { Sec- } \\
11\end{array}$ & 5 & 55 & $\mathrm{~L}$ & 50 & 50 & 6 & 250 & 564 & 9.8 & 1600 & 61 & 61 \\
\hline $\begin{array}{c}\text { Sec- } \\
10\end{array}$ & 3 & 50 & $\mathrm{~L}$ & 65 & 65 & 5 & 250 & 625 & 12.9 & 2050 & 55 & 55 \\
\hline Sec-9 & 3 & 47 & $\mathrm{~L}$ & 65 & 65 & 5 & 250 & 625 & 12.9 & 2160 & 51 & 51 \\
\hline Sec-8 & 6 & 44 & $\mathrm{~L}$ & 65 & 65 & 5 & 250 & 625 & 12.9 & 2300 & 43 & 44 \\
\hline Sec-7 & 6 & 38 & $\mathrm{~L}$ & 75 & 75 & 6 & 250 & 864 & 23.3 & 4030 & 66 & 67 \\
\hline Sec-6 & 6 & 32 & $\mathrm{~L}$ & 75 & 75 & 5 & 250 & 725 & 23.4 & 4240 & 52 & 51 \\
\hline Sec-5 & 6 & 26 & $\mathrm{~L}$ & 90 & 90 & 6 & 250 & 1044 & 28.1 & 4500 & 91 & 90 \\
\hline Sec-4 & 5 & 20 & $\mathrm{~L}$ & 75 & 75 & 6 & 250 & 864 & 23.3 & 4380 & 58 & 58 \\
\hline Sec-3 & 5 & 15 & $\mathrm{~L}$ & 90 & 90 & 6 & 250 & 1044 & 28.1 & 4670 & 86 & 86 \\
\hline Sec-2 & 5 & 10 & $\mathrm{~L}$ & 90 & 90 & 6 & 250 & 1044 & 28.1 & 4960 & 77 & 78 \\
\hline Sec-1 & 5 & 5 & $\mathrm{~L}$ & 100 & $\begin{array}{c}10 \\
0\end{array}$ & 6 & 250 & 1164 & 31.3 & 5260 & 94 & 93 \\
\hline
\end{tabular}




\section{Conclusion}

Following conclusions are drawn from the detailed analysis carried out on parametric study specifications using two different standards.

- Estimation of Wind Loads are reduced in ANSI/TIA-222H - 33\% in Square angular tower, $23 \%$ in Triangular Hybrid tower for critical wind direction. The Major Factors contributing in reduction of wind loads are.,

- Gust effect factor of 0.85 for self-supporting structures are mainly contributing on estimation of lesser wind load in American standard compared to IS 875 (Part 3).

- For Square towers, wind direction factor for corner wind is estimated using panel solidity ratio compared to standard value of 1.2 of IS 875 (Part 3)

- Force coefficient is lesser in ANSI/TIA-222H - 10\% in square tower, $5 \%$ in triangular Tower

- Force Coefficient for different MW Antenna given in ANSI Standard which lead to accurate estimation of wind load rather than generic using Indian standards

- No Major variation observed in calculation of member / connection capacity due to both standards are referring specialized code for calculation of capacities.

It is further noticed that, Tower weights approximately reduces by $10-15 \%$ based on tower configuration using ANSI/TIA-222H compared to general Indian wind loading standard (IS 875, Part 3). Therefore, specialized wind loading guidelines shall be given in Indian standard for accurate and reliable estimation of wind loads on lattice towers.

\section{References}

[1] American National Standard Institute, ANSI/TIA-222-H, Structural Standard for Antenna Supporting Structures and Antenna, Telecommunication Industry Association, October 2017.

[2] Emil Simiu, Robert H. Scanlan., Wind Effects on Structures, Fundamentals and Application to Design, Third Edition, John Wiley \& Sons, Inc. 1996.

[3] IS: 875 (Part 3) 2015 Wind Loads - Code of Practice for Design Loads (Other than Earthquake) for Buildings and structures.

[4] N. Subramanian, Design of Steel Structures, Oxford University Press, India, 2008.

[5] Robert H. Durfee., Review of Triangular Cross Section Truss Systems, Journal of Structural Engineering, Vol. 112, No.5, May 1986.

[6] Venkateswarlu. B., Hari Krishna, P., Selvi Rajan, S., Satish Ram Kumar, M., Stochastic Gust Response of Microwave Lattice Towers, Computers \& Structures, Vol.52, No.5, pp 1031-1041, 1994. 